\title{
A potential lag between the open solar magnetic source flux and solar EUV and X-ray emissions as measured by the Earth's ionosphere during total solar eclipses
}

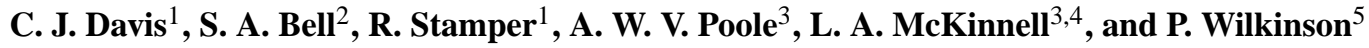 \\ ${ }^{1}$ Rutherford Appleton Laboratory, Chilton, Oxfordshire, OX11 0QX, UK \\ ${ }^{2}$ HM Nautical Almanac Office, UK Hydrological Office, Admiralty Way, Taunton, TA1 2DN, UK \\ ${ }^{3}$ Department of Physics and Electronics, Rhodes University, Grahamstown, South Africa \\ ${ }^{4}$ Hermanus Magnetic Observatory, Hermanus, South Africa \\ ${ }^{5}$ IPS Radio and Space Services, Haymarket, NSW 1240, Australia
}

Received: 30 March 2009 - Revised: 5 June 2009 - Accepted: 8 June 2009 - Published: 18 June 2009

\begin{abstract}
Measurements of the ionospheric E-region during total solar eclipses have been used to provide information about the evolution of the solar magnetic field and EUV and $\mathrm{X}$-ray emissions from the solar corona and chromosphere. By measuring levels of ionisation during an eclipse and comparing these measurements with an estimate of the unperturbed ionisation levels (such as those made during a control day, where available) it is possible to estimate the percentage of ionising radiation being emitted by the solar corona and chromosphere. Previously unpublished data from the two eclipses presented here are particularly valuable as they provide information that supplements the data published to date. The eclipse of 23 October 1976 over Australia provides information in a data gap that would otherwise have spanned the years 1966 to 1991 . The eclipse of 4 December 2002 over Southern Africa is important as it extends the published sequence of measurements. Comparing measurements from eclipses between 1932 and 2002 with the solar magnetic source flux reveals that changes in the solar EUV and X-ray flux lag the open source flux measurements by approximately 1.5 years. We suggest that this unexpected result comes about from changes to the relative size of the limb corona between eclipses, with the lag representing the time taken to populate the coronal field with plasma hot enough to emit the EUV and X-rays ionising our atmosphere.
\end{abstract}

Keywords. Ionosphere (Solar radiation and cosmic ray effects) - Solar physics, astrophysics, and astronomy (Corona and transition region; Magnetic fields)

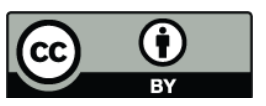

Correspondence to: C. J. Davis (chris.davis@stfc.ac.uk)

\section{Introduction}

Measurements of EUV and X-ray emissions from the solar corona and chromosphere as measured by the response of the Earth's ionosphere during total solar eclipses have been presented in a previous publication (Davis et al., 2001). This work was carried out to obtain information about the long-term changes in these emissions in order to supplement work that indicated that the strength of the coronal magnetic source flux had doubled over the last century (Lockwood et al., 1999). As details of the technique are contained in the previous paper (Davis et al., 2000), only a brief overview of the method will be presented here.

By measuring the variation of electron concentration in the Earth's ionospheric E-region during an eclipse and comparing these measurements with similar unperturbed ionisation levels (such as those made during a control day, where available) it is possible to estimate the fraction of ionising radiation, $\Phi$, being emitted by the solar corona and chromosphere

$\Phi=\frac{d N_{E} / d t+\alpha N_{E}^{2}}{d N_{C} / d t+\alpha N_{C}^{2}}$

where $N_{E}$ and $N_{C}$ are the electron concentration on the day of the eclipse, and the control day respectively and $\alpha$ is the recombination rate for the ionisation at E-region altitudes (around $100 \mathrm{~km}$ ).

In order that values of $\Phi$ can be compared between eclipses, these values are corrected to allow for the apparent diameter of the lunar shadow with respect to the solar disk, as this differs between eclipses. Davis et al. (2001) identified two methods of correction. Firstly a simple geometric

Published by Copernicus Publications on behalf of the European Geosciences Union. 


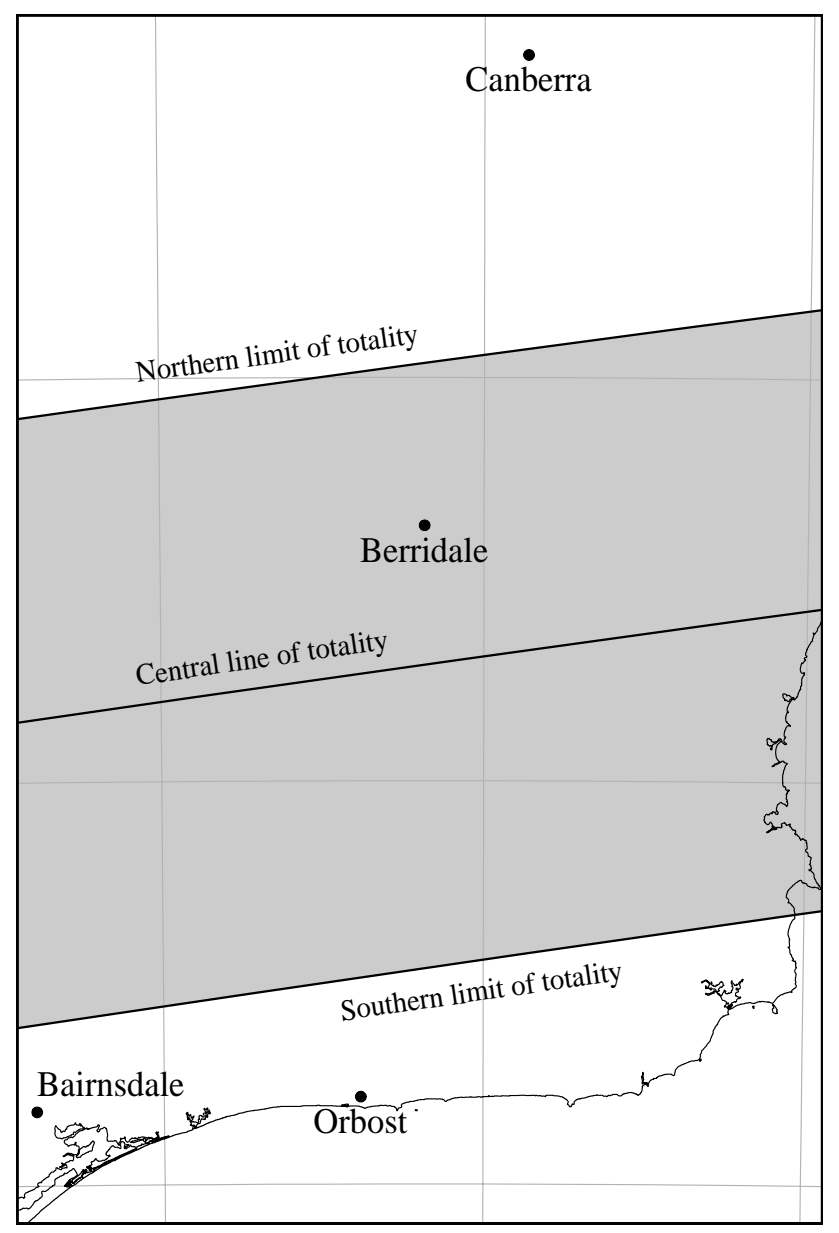

Fig. 1. The path of totality at an altitude of $100 \mathrm{~km}$ for the solar eclipse over Berridale, Australia on 23 October 1976.

correction that assumes a constant emission level for the entire chromosphere and corona;

$\Phi^{\prime}=1-(1-\Phi)\left(\frac{r}{r+d r}\right)^{2}$

where $r$ is the apparent solar radius and $r+d r$ is the apparent lunar radius. Secondly by applying a more detailed correction that uses estimates of the average coronal emission as a function of solar radius (as estimated from Solar Heliospheric Observatory, $\mathrm{SOHO}$, data) to produce a corrected value, $\Phi_{S}$. Two such functions were produced, for solar maximum and minimum. Values of $\Phi^{\prime}$ could therefore be estimated for both solar maximum and solar minimum conditions. Assuming that the variation between these two profiles is linear, this gives;

$\Phi_{S}=f \Phi_{S \max }+(1-f) \Phi_{S \min }$

where $f$ is the fractional phase of the solar cycle on the day of the eclipse. This fractional phase was characterised for each eclipse by calculating the sunspot number on that date as a fraction of the range of sunspot numbers in that cycle. As such, it represents a fraction of the activity within that cycle rather than the fraction of the cycle duration. While such a definition is free from uncertainties introduced by the variable nature of the length and shape of each activity cycle, it remains important to ascertain how sensitive the resulting correction factor is to systematic errors in the chosen value of $f$. In order to investigate this, the sensitivity of the correction factor was calculated as a function of $f$ and $(r+d r) / r$, the ratio of lunar to solar radii. It was found that, for all radii ratios between 1 and 1.15, a $10 \%$ uncertainty in fintroduced an uncertainty into the correction factor of no more than $2 \%$. It should be noted that Eq. (3) represents the corrected form of the equation quoted in Davis et al. (2001) (a typographical error that does not affect their results or conclusions). While the assumption of a linear variation in this function is simplistic, we use this method for consistency with results published previously. The limb profiles used were originally derived from SOHO data prior to 2001 and so a more detailed study can now be made of this variation. This will be the subject of future work.

\section{Previously unpublished results}

\subsection{October 1976}

This eclipse was observed from Berridale, Australia $\left(36^{\circ} 22^{\prime} \mathrm{S}, 148^{\circ} 49^{\prime} \mathrm{E}\right)$ using an IPS 4B ionosonde. Results were recorded to and scaled from $16 \mathrm{~mm}$ film. In the E-region, at an altitude of $100 \mathrm{~km}$, the eclipse began at 05:40:19 UT and ended at 07:44:51 UT with two and a half minutes of totality between 06:44:27 UT and 06:46:57 UT. A map of the path of totality at $100 \mathrm{~km}$ is shown in Fig. 1. The ionograms for this day contained sporadic-E reflections that, in some cases, obscured or introduced uncertainty into the scaled $f o$ E values. Where Es obscured the E-layer values, the data were discarded. This accounts for the brief data gap between 15:30 and 16:00 UT. Fortunately there was no such ambiguity at totality. No control data were available and so a polynomial was fitted to the data prior to the onset of the eclipse on the 23 October and this was used to estimate the unperturbed ionospheric variation during the eclipse. Since the eclipse occurred in the latter half of the day, this provided nine hours of data for the fit.

Figure 2a shows the variation in electron concentration (as estimated from $f o \mathrm{E}$ values) for 23 October 1976 compared with the polynomial used to estimate the non-eclipsed ionospheric variation in the absence of control data. By comparing these two data sets, $\Phi$ can be estimated from Eq. (1) and these results are shown in Fig. 2b. For Berridale, $\Phi_{\min }=10.9 \pm 0.7$. By applying the two correction factors described previously, $\Phi^{\prime}=18.7 \pm 1.2$ and $\Phi_{S}=17.1 \pm 4.1$. 

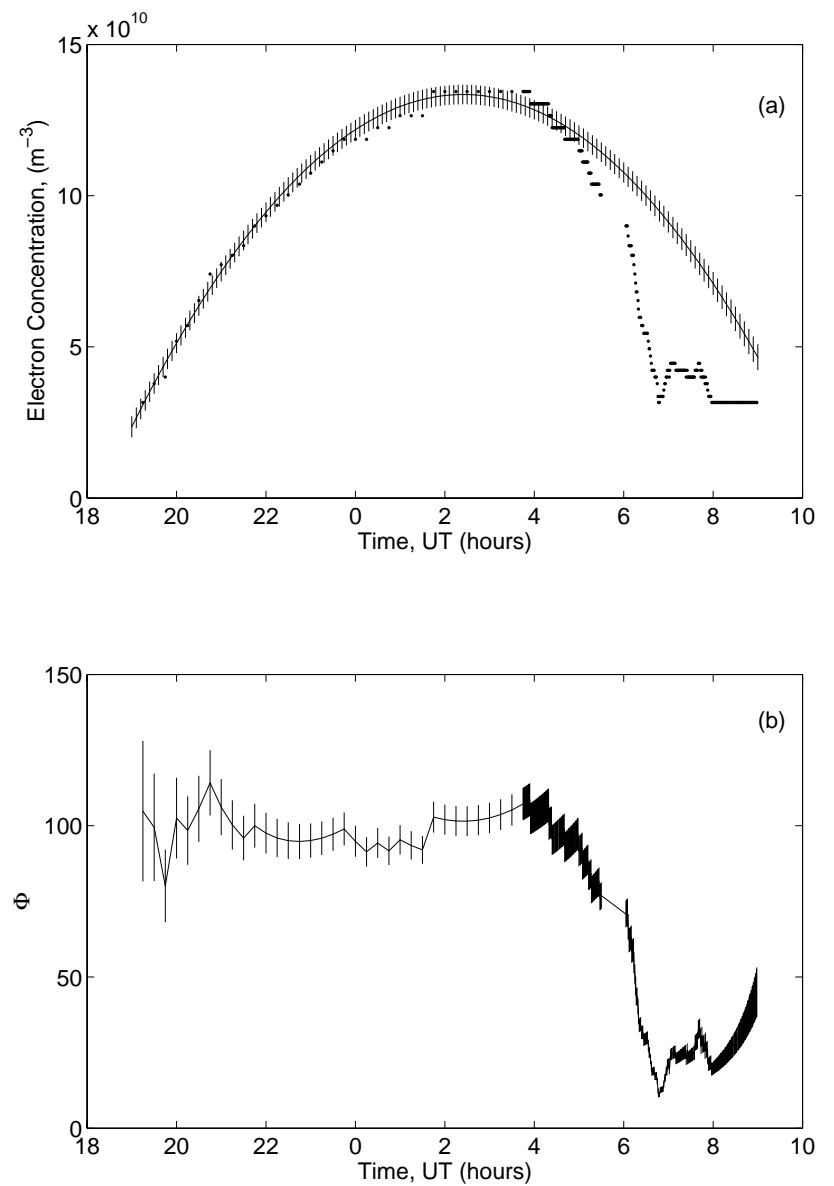

Fig. 2. (a) Electron concentration in the ionospheric E region during eclipse at Berridale (data points) compared with the control data (solid curve). For this eclipse, the control data were obtained by fitting a parabola to the data from 23 October prior to the onset of the eclipse. (b) The fraction of ionising radiation, $\Phi$, visible during the total eclipse at Berridale on 23 October 1976.

\subsection{December 2002}

This eclipse was observed from Madimbo, South Africa $\left(22^{\circ} 22.8^{\prime} \mathrm{S}, 30^{\circ} 52.8^{\prime} \mathrm{E}\right)$ using a Lowell Digisonde DPS-4 (Bibl and Reinisch, 1978). Results were recorded digitally and manually edited using the software provided. At an altitude of $100 \mathrm{~km}$ above this location, the eclipse began at 05:11:02 UT and ended at 07:35:44 UT with one minute and twenty seconds of totality between 06:17:52 UT and 06:19:12 UT. The path of totality at $100 \mathrm{~km}$ is shown in Fig. 3. Control data were provided by measurements from the previous day, 3 December 2002.

Figure 4 a shows the variation in electron concentration (as estimated from $f o \mathrm{E}$ values) for 4 December 2002 compared with the polynomial fitted to the control data (taken from the previous day, 3 December 2002). The resulting values of $\Phi$ are shown in Fig. 4b. For this eclipse, $\Phi_{\min }=13.5 \pm 1.1$ and after corrections have been applied, $\Phi^{\prime}=17.0 \pm 1.4$ and

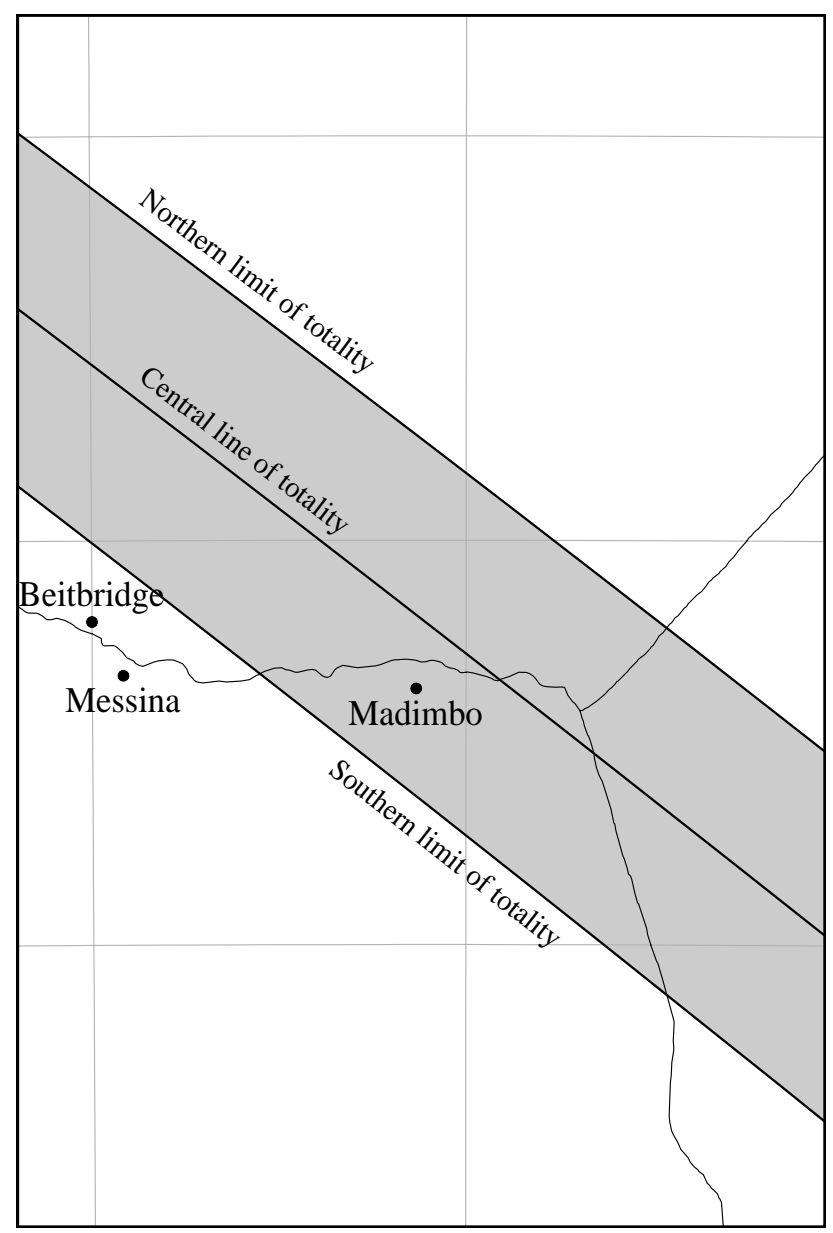

Fig. 3. The path of totality at an altitude of $100 \mathrm{~km}$ for the solar eclipse over Madimbo, South Africa, on 4 December 2002.

$\Phi_{S}=18.2 \pm 4.5$ (with a sunspot number of 82 in December 2002 , in a cycle with a range of 169 , the estimated value for $f$ was 0.48 ).

\section{Eclipse observations and the coronal magnetic source flux}

When the two additional eclipse results are plotted alongside the previously evaluated estimates for $\Phi, \Phi^{\prime}$ and $\Phi_{S}$ for all other eclipse observations (Figs. 5, 6 and 7) it is clear that estimates from these two dates provide valuable additional information both during a previously large data gap and the first estimate after the peak of the last cycle when the source flux itself is seen to be in decline. While there appears to be little relation between the uncorrected $\Phi$ values and the coronal source flux (Fig. 5), when correction is made for the apparent size of the Moon, the resulting $\Phi^{\prime}$ values appear to follow the changes in coronal source flux more closely (Fig. 6). A similar correlation is seen when the $\Phi$ values are 

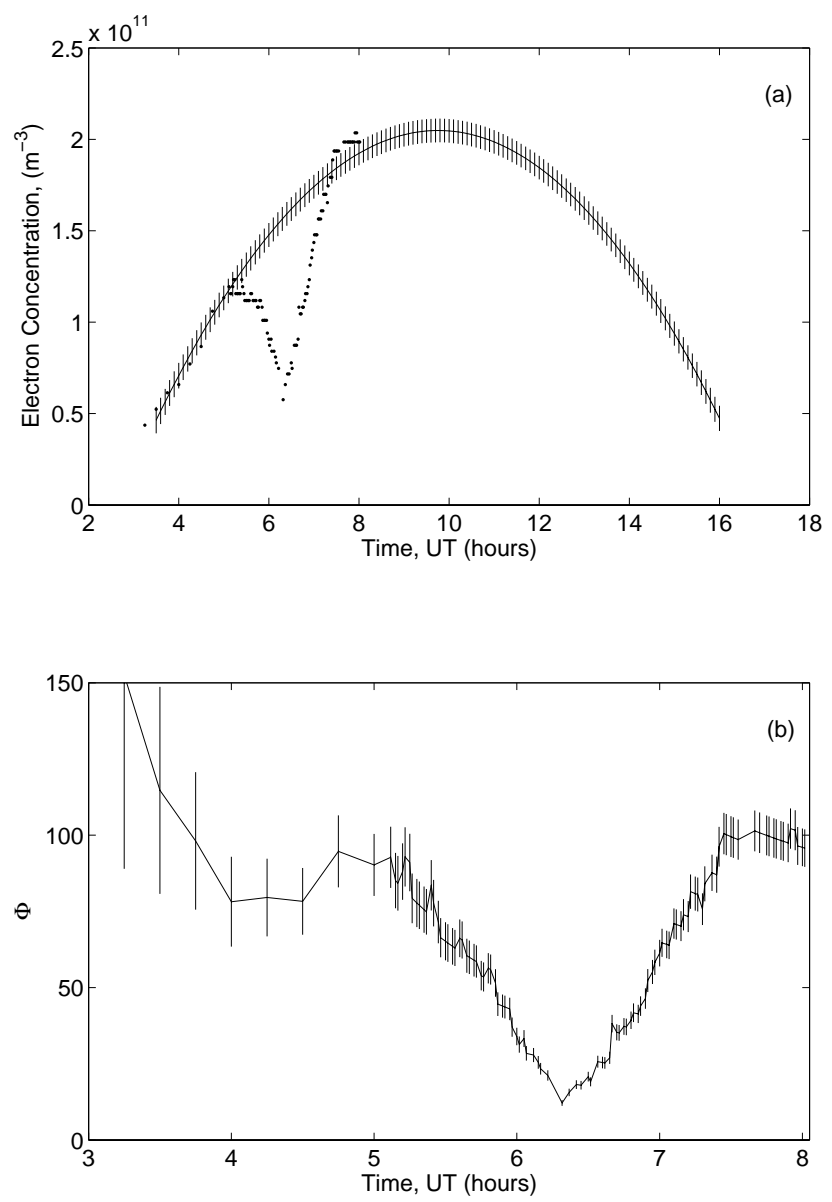

Fig. 4. (a) Electron concentration in the ionospheric E-region during eclipse at Madimbo, South Africa (data points) compared with the control data (solid curve). For this eclipse, the control data were obtained by fitting a parabola to data scaled from the previous day. (b) fraction of ionising radiation, $\Phi$, visible during the total eclipse at Madimbo, South Africa, on 4 December 2002.

corrected using an estimate of the coronal limb profile from SOHO data, $\Phi_{\mathrm{S}}$ (Fig. 7).

It is not surprising that trends in $\Phi$ show similar variations to the coronal source flux since both are influenced by the evolution of the solar corona. $\Phi$ values represent the fraction of ionising radiation emitted by plasma in the uneclipsed corona. Since the apparent area of the eclipsed disk will remain relatively constant as viewed from Earth, an increase in $\Phi$ represents an increase in the size of the extended corona with respect to this disk. Similarly, the coronal source flux is a measure of magnetic field threading the solar source surface and extending into the heliosphere. An increase in coronal source flux therefore represents an expansion of coronal magnetic fields across this surface. As these field lines are populated with plasma, this will modulate the distribution of ionising radiation. The correlation between these two data sets, together with any observed lag, therefore provides in-

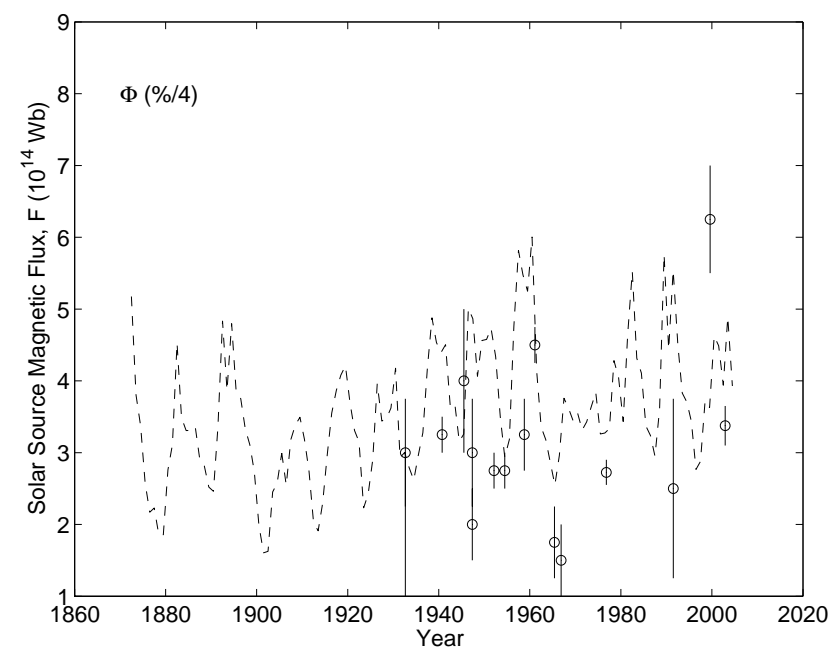

Fig. 5. A comparison of uncorrected $\Phi$ values obtained for 15 eclipses from 1932 to 2002 compared with the total solar source flux, $F$.

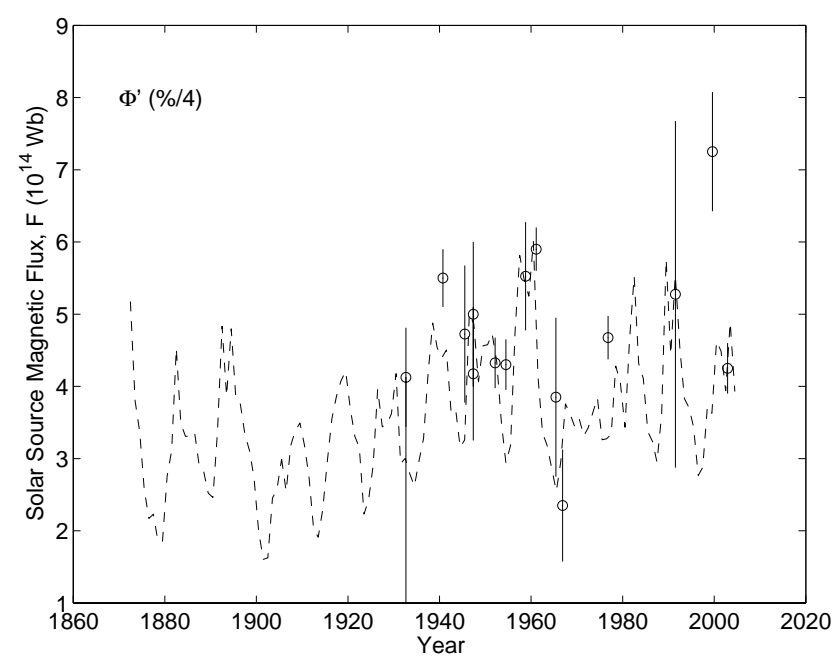

Fig. 6. The same as Fig. 5 but after the $\Phi$ values have been corrected for the relative size of the lunar shadow assuming a linear change in coronal intensity with radius to give $\Phi^{\prime}$ values.

formation about the timescales of the mechanisms involved in the evolution of the solar magnetic field.

In this analysis, we are comparing yearly source flux averages with measurements of EUV and X-ray emissions from the limb-corona made over a few hours. Given this difference, it is perhaps surprising that so many of the eclipse measurements follow the source flux trend so well. Such a correlation could be taken to indicate that the underlying intensity of EUV and X-ray emissions from the Sun vary over much longer timescales than a day. Any deviation from this agreement could be due to a short-lived event such as a solar flare or coronal mass ejection which adds to the background emission intensity. 


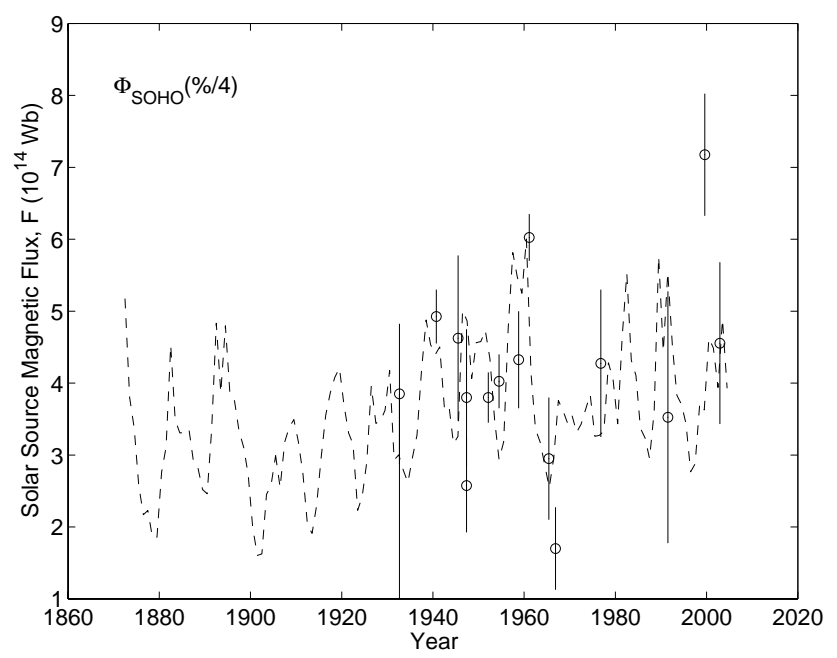

Fig. 7. The same as Fig. 5 but after the $\Phi$ values have been corrected for the relative size of the lunar shadow using an intensity function based on observations of the average coronal intensity measured by the SOHO spacecraft to give $\Phi_{S}$.

\section{Correlating the two data sets}

The following correlations were carried out using $\Phi^{\prime}$ values since some correction is necessary to account for the small variation in apparent lunar diameter between eclipses. For completeness, the results of a similar analysis using $\Phi_{\mathrm{SOHO}}$ corrected values is summarised at the end of this section.

A Pearson's correlation test was applied to the two datasets for a range of lags. The significance of the correlation was then calculated from the Student's t statistic;

$t=|c c|\left\{\frac{n_{i}-2}{1-|c c|}\right\}^{\frac{1}{2}}$

Where $c c$ is the correlation coefficient and $n_{i}$ is the number of independent samples computed from the number of data points $\mathrm{n}$, allowing for persistence in the data. To account for persistence in the data, the first lag in the auto-correlation functions (ACFs) of both data sets $(\alpha 1, \alpha 2)$ was used to estimate the number of independent data points $\left(n_{i}\right)$ from the expression;

$n_{i}=n\left(1-\alpha_{1} \alpha_{2}\right) /\left(1+\alpha_{1} \alpha_{2}\right)$

where $n$ is the number of eclipse observations. Having calculated the $\mathrm{t}$ statistic, the significance can be obtained from the $t$ cumulative distribution function, with the number of degrees of freedom $n_{i}-2$.

The ACF of the coronal source flux data gives a value at lag 1 of 0.72 (when the zeroth lag is scaled to unity). The equivalent for the sequence of eclipse observations is not so straight forward since the data are not evenly sampled in time. An estimate of this lag value can be obtained by using the Discrete Correlation Function (DCF) (Edelson and
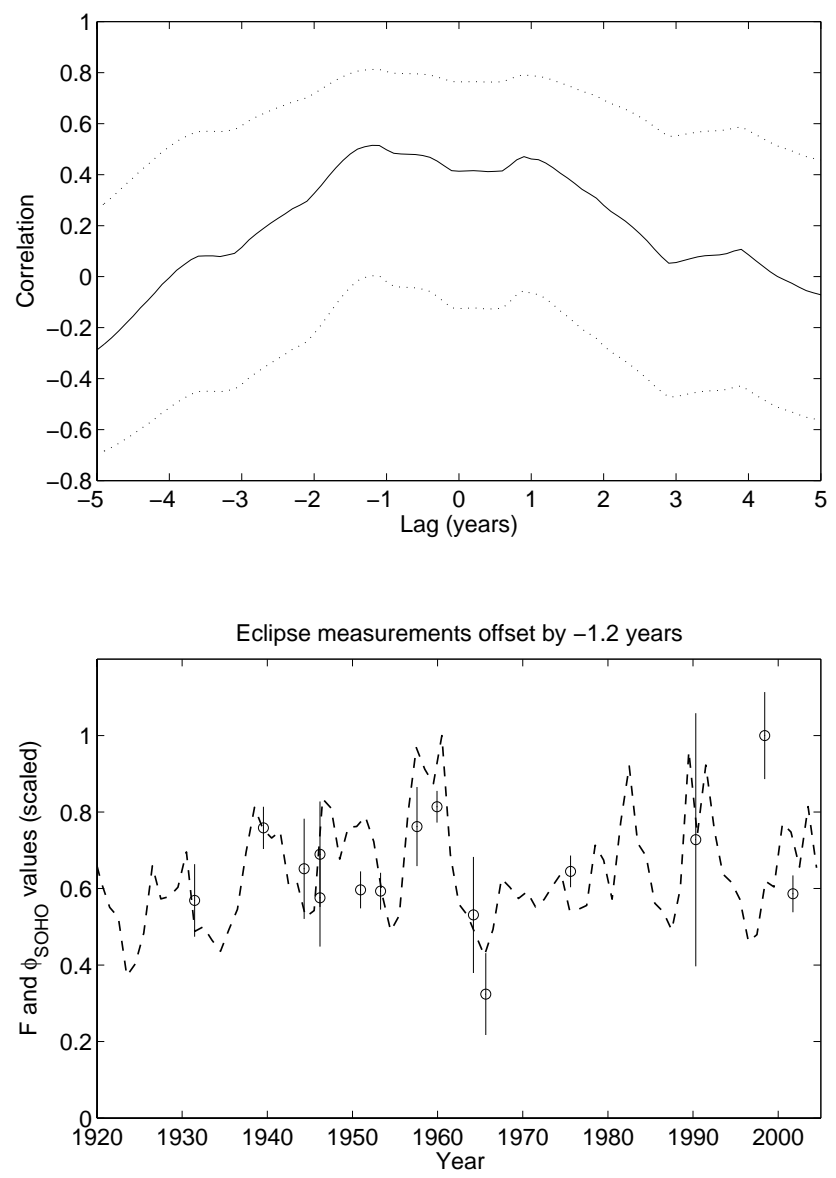

Fig. 8. (a) Variation of correlation coefficient between values of $\Phi_{\min }$ and solar magnetic source flux for lags between -5 and 5 years (solid line). The dotted lines represent the upper and lower bounds of the $95 \%$ confidence interval for each coefficient. (b) Solar source flux compared with the same $\Phi^{\prime}$ values as shown in Fig. 6 but now with the eclipse measurements offset by -1.2 years where the maximum correlation between the two data sets occurs.

Krolik, 1988). When applied to the time series of eclipse observations, this gives a value for the first lag of 0.46 for $\Phi_{\min }$ values, 0.8 for $\Phi^{\prime}$ values and 0.37 when applied to $\Phi_{\mathrm{SOHO}}$ values.

For each lag in the range -5 to +5 years, coronal source flux values at the times of the eclipse observations were estimated by interpolation. It was found that a maximum correlation of 51\% occurred for $\Phi^{\prime}$ values when they were offset by $-1.2_{-0.4}^{+2.4}$ years compared with the source flux (Fig. 8). The uncertainties represent the range of lag values where the probability of the correlation occurring by chance was within $5 \%$ of the peak lag value. The significance of this result is not great, with a $19 \%$ probability that it occurred by chance.

With so few (15) data points, the chances of a single point having a disproportionate influence on such a correlation becomes significant. In order to investigate whether any of the individual eclipse measurements were having 

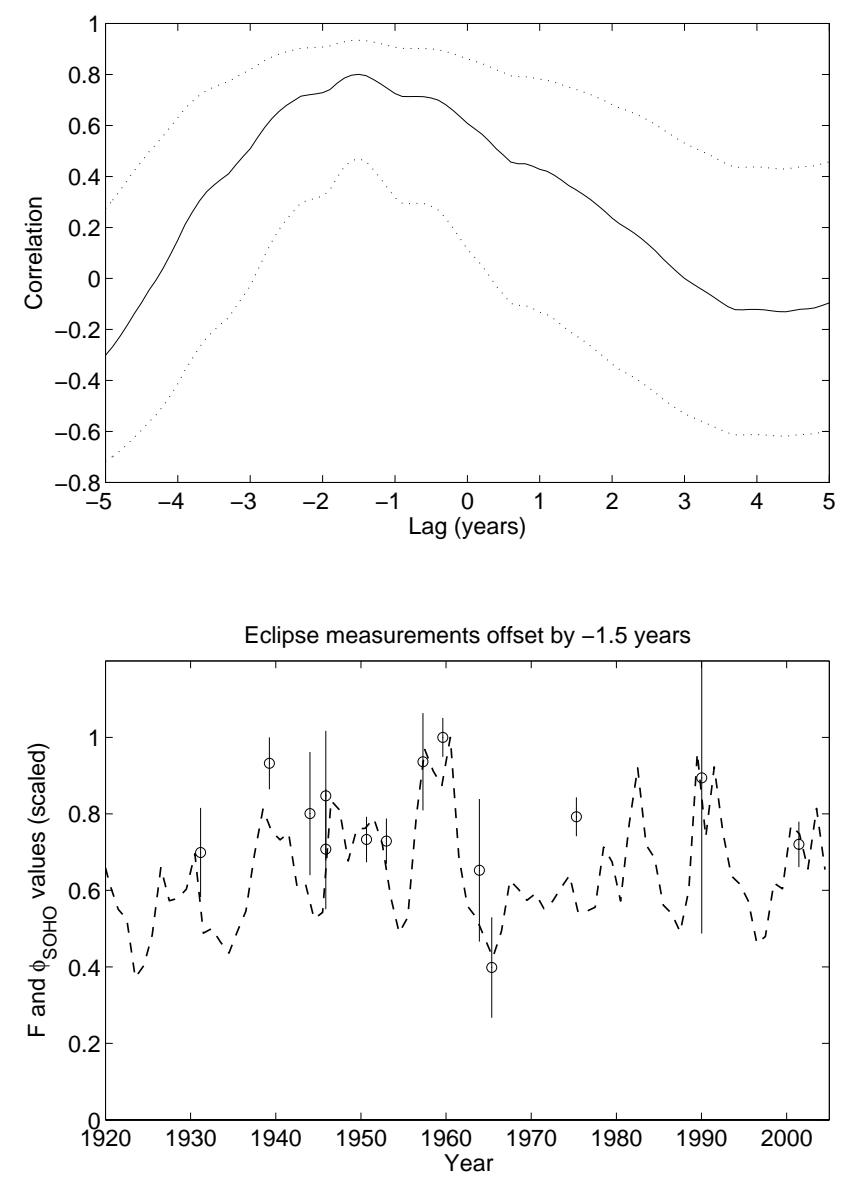

Fig. 9. (a) Variation of correlation coefficient between 14 values of $\Phi^{\prime}$ and solar magnetic source flux for lags between -5 and 5 years (solid line). The dotted lines represent the upper and lower bounds of the $95 \%$ confidence interval for each coefficient. (b) Solar source flux compared with 14 of the previous $\Phi^{\prime}$ values (an outlier having been identified and removed) offset by -1.5 years corresponding to the offset where the maximum correlation between the two data sets occurs.

an unreasonably large influence on the correlation process, Cook's distance (Cook, 1979) was calculated for all points at each lag. This parameter is a measure of the influence each data point has on all the other data in a fit. A data point is considered to have an unreasonably large influence over the rest of the data set if its Cook's distance is greater than the median of the F-distribution. When the eclipse data were examined in this way, it was found that one data point was having a significant influence over the rest of the data, particularly at longer lags. This point is the observation made during the solar eclipse in 1999 for which Cook's distance exceeded the threshold by over $30 \%$.

If this data point is discarded and the correlation recalculated, a maximum correlation of $80 \%$ occurs when the $\Phi$ ' eclipse measurements are offset by $-1.5_{-1.4}^{+1.9}$ years (Fig. 9). The significance of this correlation, once again taking the
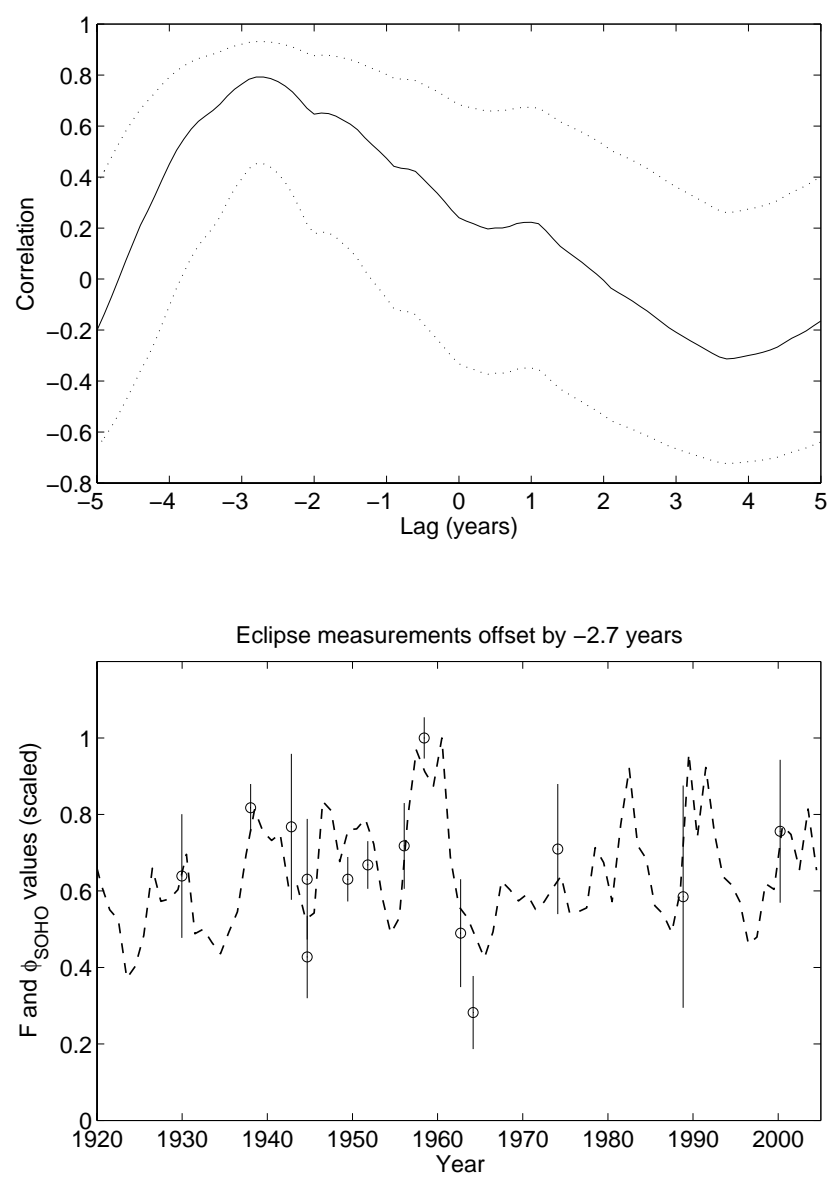

Fig. 10. (a) Variation of correlation coefficient between 14 values of $\Phi_{S}$ and solar magnetic source flux for lags between -5 and 5 years (solid line). The dotted lines represent the upper and lower bounds of the $95 \%$ confidence interval for each coefficient. (b) Solar source flux compared with $14 \Phi$ values corrected using a coronal limb profile calculated from SOHO data. These $\Phi_{\mathrm{S}}$ data are offset by -2.7 years corresponding to the maximum correlation between these two data sets.

persistence in the two data sets into account, is $96 \%$ (a $4 \%$ likelihood that the correlation occurred by chance).

While this revised result appears to be an improvement, before we discard a data point, it is important to understand why that data point may be inconsistent with the rest of the measurements.

There were no significant changes in solar emissions or solar activity during the eclipse of 11 August 1999. The aa index, from which the solar source flux estimate is derived, is only 14.1 on that day and does not vary significantly around that date. It is likely therefore that the apparent inconsistency of this result lies in the fact that the observations made at Helston in Cornwall in 1999 were made at the extreme northern limit of the path of totality at $100 \mathrm{~km}$ (Davis et al., 2000). It was estimated that totality lasted around $8 \mathrm{~s}$ while the cadence of the observations was of the order of one minute. It is 
conceivable therefore that the true minimum value occurred between measurements and that the recorded minimum ionospheric concentration represents an upper limit only.

The above analysis was carried out with the values of $\Phi^{\prime}$ observations corrected using a geometrical factor to account for the relative size of the Moon with respect to the Sun. When the analysis was repeated for uncorrected $\Phi$ values, a maximum correlation of 0.68 significant to $98 \%$ was found when the eclipse observations were offset by $-2.8_{-1.1}^{+1.5}$ years. For those data scaled using a limb correction derived from SOHO data, $\Phi_{\mathrm{S}}$, a maximum correlation of 0.79 (significant to $99 \%$ ) was found when the eclipse observations were offset by $-2.7_{-1.1}^{+1.5}$ years (Fig. 10). While the various estimates of the offset between the eclipse measurements and the source flux may differ in value and significance, they are consistent in indicating that the coronal emission intensity (as measured by the ionospheric response during total solar eclipses) appears to lag the evolution of the solar coronal field and this has interesting implications for the evolution of the solar corona.

\section{What could such a lag indicate?}

The presence of a potential lag between the source flux values and the eclipse is curious when considered in the context of other observations and modelling work. There is evidence, for example, that the cosmic ray flux observed at Earth lags solar activity by several months (Minarovjech and Kudela, 2004). Since the cosmic ray flux arriving at Earth is modulated by the interplanetary magnetic field, this could be interpreted as evidence that the emergence of magnetic flux across the source surface lags solar activity. In their study Minarovjech and Kudela estimated solar activity from both sunspot number and the coronal green line index and obtained similar results for both when comparing them with cosmic ray data. Modelling work on the evolution of the coronal magnetic field during the solar cycle (Wang and Sheeley, 2003) indicates that the time taken for emerging loop structures to propagate outwards and polewards from the photospheric equator and populate the far corona is of the order of years. Open flux accounts for $1-2 \%$ of the magnetic flux emerging from the photosphere. Once it has reached the source surface, changes to the open flux propagate rapidly through the heliosphere, reaching $1 \mathrm{AU}$ in timescales of the order of a few days. Since coronal emissions result from plasma associated with these magnetic structures, it would therefore seem reasonable to suppose that, if anything, measurements of the coronal source flux at Earth would lag the intensity of coronal emissions. To investigate this we cross-correlated the coronal green line index with the coronal source flux. While the maximum correlation (allowing for persistence in the data) was 0.74 with a significance of 0.97 , there was no significant time lag between the two data sets $\left(0.1_{-2.0}^{+3.4}\right)$.
To ensure that the variation we see in $\Phi^{\prime}$ is not simply due to changes in the emission intensity of the corona, we compared the coronal green line index with the eclipse measurements. If there is a significant offset in time between the direct measure of the coronal intensity and those inferred from the eclipse measurements, this is evidence that the observed changes in $\Phi^{\prime}$ result from something other than a change in total intensity.

Taking monthly values of the coronal green line index and comparing them with the relative intensities corrected for geometric effects, $\Phi^{\prime}$, gives a maximum correlation of 0.7 (significant to $96 \%$ when the persistence of the two data sets are accounted for) when the eclipse measurements are offset by $-1.8_{-1.4}^{+1.7}$ years with respect to the coronal index. This is consistent with our previous results.

\section{Discussion and conclusion}

In order to interpret our results, it is useful to recall that with the eclipse measurements, $\Phi$, represents the intensity of the uneclipsed fraction of the corona compared with emission from the entire Sun. If the emission from the corona and disk both increased in intensity, then the ratio we are measuring would not change. If the location of emission changes, with a greater proportion coming from higher in the corona where it is not obscured during an eclipse, the value of $\Phi$ will increase. As a result, changes in $\Phi$ between eclipses indicates a redistribution of the emission rather than changes in total intensity. Such changes in total intensity may occur but our technique does not provide any information on them.

Since we have a direct observation of changes in the coronal green line emission intensity and these are lagged by the eclipse measurements, it seems likely that the trends in the two data sets result from two different causes (although it must be remembered that the coronal green line is only part of the spectrum of solar radiation responsible for ionising the Earth's upper atmosphere and may not be representative of the whole spectrum).

Why do the changes in distribution of the ionisation appear to lag the emission intensity and the solar source flux? Does this time lag represent the time necessary to populate expanded field lines with plasma or the time required to heat such plasma to temperatures necessary to generate EUV and $\mathrm{X}$-ray radiation? This latter point could be tested by studying the total intensity and distribution of such radiation throughout the entire solar cycle as measured by spacecraft such as SOHO. We have embarked on such a study which we intend to present in a future publication.

Observations of the ionospheric response to solar eclipses are still somewhat limited as they either occur through serendipitous alignments or as a result of special campaigns that require much time and effort. Adding data from future eclipse experiments or previously unpublished results is 
required to improve this result and the authors encourage others to carry out such observations when possible.

Acknowledgements. C. J. Davis would like to thank A. Rouillard and M. Lockwood for provision of the coronal source flux data and for useful discussions.

Topical Editor R. Forsyth thanks two anonymous referees for their help in evaluating this paper.

\section{References}

Bibl, K. and Reinisch, B. W.: The universal digital ionosonde, Radio Sci., 13(3), 519-530, 1978.

Cook, R. D.: Influential observations in linear regression, J. Am. Statist. Assoc., 74, 169-174, 1979.

Davis, C. J., Lockwood, M., Bell, S. A., Smith, J. A., and Clarke, E. M.: Ionospheric measurements of relative coronal brightness during the total solar eclipses of 11 August, 1999 and 9 July, 1945, Ann. Geophys., 18, 182-190, 2000,

http://www.ann-geophys.net/18/182/2000/.
Davis, C. J., Clarke, E. M., Bamford, R. A., Lockwood, M., and Bell, S. A.: Long term changes in EUV and X-ray emissions from the solar corona and chromosphere as measured by the response of the Earths ionosphere during total solar eclipses from 1932 to 1999, Ann. Geophys., 19, 263-273, 2001, http://www.ann-geophys.net/19/263/2001/.

Edelson, R. A. and Krolik, J. H.: The Discrete Correlation Funciton - A new method for analyzing unevenly sampled variability data, Astrophys. J., 333, 646-659, 1988.

Lockwood, M., Stamper, R., and Wild, M. N.: A doubling of the Sun's coronal magnetic field during this century. Nature, 399(6735), 437-439, 1999.

Minarovjech, M. and Kudela, K.: Cosmic rays and solar coronal emissions: cross-correlations of the time series, Solar Phys., 224, 285-290, 2004.

Wang, Y.-M. and Sheeley Jr., N. R.: On the topological evolution of the coronal magnetic field during the solar cycle, The Astrophys. J., 599, 1404-1417, 2003. 